\title{
EL PODER Y LA GESTIÓN DEL CONOCIMIENTO: LAS CLAVES DEL CAMBIO ORGANIZACIONAL
}

Autores: Álvaro Enrique Santamaría Escobar (1)

Wilson Cadrazco Parra (2)

\section{RESUMEN}

-1 1 poder en sus diferentes manifestaciones, y el conocimiento, han cobrado vigencia en medio del esfuerzo que haA cen las organizaciones por desarrollar un cambio organizacional planeado. Debido a la revitalización que estos dos conceptos han tenido en el ámbito de la gestión estratégica de las organizaciones, la presente revisión tiene por objetivo, identificar y analizar las principales tendencias derivadas del rol del poder y la gestión del conocimiento en el cambio organizacional. Se concluye que el conocimiento y el poder están transformando a la configuración organizacional, sus productos, sus procesos, e incluso su mercado.

Palabras claves: Cambio, Poder Y Conocimiento

\section{ABSTRACT}

$\mathrm{P}$ ower in its various manifestations and knowledge, have reemerged amid efforts made by organizations to develop a planned organizational change. Due to the revitalization that these two concepts in the field of strategic management of organizations, the present review aims to identify and analyze the main tendencies arising from the role of power and knowledge management in organizational change. We conclude that knowledge and power are transforming the organizational configuration, its products, processes, and even their market .

Keywords: Change, Power And Knowledge .

(1) Álvaro Enrique Santamaría Escobar. Ingeniero Industrial, Docente de Planta de la Universidad de Sucre - Colombia.

(2) Wilson Cadrazco Parra. Administrador de Empresas. Docente de Planta de la Universidad de Sucre. 
EL PODER Y LA GESTIÓN DEL CONOCIMIENTO: LAS CLAVES DEL CAMBIO ORGANIZACIONAL

\section{INTRODUCCIÓN}

L as investigaciones que relacionan poder, cambio organizacional y gestión del conocimiento $\mathrm{KM}$, han tomado auge en las últimas décadas, autores como (Thomas, Sargent, y Hardy, 2011; Perry, Snunith, 2009; Li, Zhang, J., Zhang, X., 2013; Sanchez, Mahoney, 1996); han incursionado en este campo, y coinciden en la relevancia que tiene la gestión del conocimiento para el propósito del cambio organizacional. Además algunos investigadores relacionan la $\mathrm{KM}$, el cambio organizacional, y el poder ( ), en suma el abordaje en el campo está mostrando novedosas oportunidades para consolidar la estrategia y la configuración empresarial.

El objetivo de esta revisión consiste en identificar y analizar las principales tendencias derivadas del rol del poder y la gestión del conocimiento en el cambio organizacional.

Inicialmente muestra a una aproximación conceptual relacionada al cambio, el poder y la gestión el conocimiento. En un segundo apartado aborda el rol del poder y la gestión del conocimiento en el cambio organizacional, y finalmente se concluye.

\section{DESARROLLO}

A ntes de entrar en el entramado de estos tres constructos, es importante contextualizar cada uno de ellos en el ámbito organizacional.

Para Robbins y Judge (2013a) el cambio es "toda acción que implica realizar las actividades de manera diferente" (p.581). En materia organizacional, el cambio no se presenta de forma espontanea, por el contrario, el cambio es un factor clave para la desarrollar ventajas competitivas; En este sentido, éstos autores introducen el concepto del cambio planeado, el cual en las organizaciones está relacionado con la estrategia, y "corresponde a actividades que son intencionales y están orientadas a alguna meta" (Robbins, Judge, 2013b, p. 581).

Debido al acelerado cambio reinante en la economía global, las organizaciones exitosas, hoy deben fomentar las innovaciones y dominar el arte del cambio o se volverán candidatas a la extinción. En ese sentido, los empleados de la organización pueden ser el impulso para la innovación o el cambio o bien el mayor escollo (Robbins, 1998c, p.12).

Zapata et al. (2009a) asocia el poder y la cultura organizacional, toda vez que el ejercicio del poder es un reflejo de la manera como se hacen las cosas en el seno de las organizaciones. En ese sentido, menciona que existe una fuerte influencia sobre algunas de las dimensiones de la cultura corporativa, como lo son: el proceso de toma de decisiones, el diseño y rediseño de normas, de leyes y de la estructura organizacional, las relaciones intergrupales, el liderazgo y el uso de autoridad entre otros ( $p$. 207).

Es de anotar entonces, que el poder contribuye a moldear la cultura organizacional, y por tanto es un factor facilitante del cambio, sin embargo, en este aspecto, es importante reconocer que los intereses de los individuos, no siempre van de la mano con los intereses de la organización. Esta situación socava la cohesión interna de la organización, genera conflicto y resistencia, y debilita la cultura organizacional.

Debido a que los individuos en las organizaciones tienen sus propios intereses, las empresas no concentran todos sus esfuerzos en optimizar los beneficios. Los miembros de estas organizaciones negocian o desarrollan contrapartidas políticas entre sí para llegar a definir un conjunto de objetivos más o menos aceptables para todos (Whittington, 2002), por lo que las empresas suelen tener suficiente poder de mercado como para obtener beneficios razonables sin realizar su máximo esfuerzo. 
EL PODER Y LA GESTIÓN DEL CONOCIMIENTO: LAS CLAVES DEL CAMBIO ORGANIZACIONAL

En los párrafos anteriores se hizo alusión al cambio y al poder, y se observo la estrecha relación de estos constructos con la cultura organizacional, a continuación se introduce el concepto de gestión del conocimiento, el se ha convertido en un factor determinante para la transformación de las organizaciones en organizaciones inteligentes, generadoras de valor.

Antes de involucrar la gestión del conocimiento, como elemento fundamental en este campo, es importante conocer antes acerca del concepto de conocimiento, (Argyris, 1999; Sveiby, 2000) definen el conocimiento como una capacidad de actuación de cada persona, que se va creando de forma continuada con el aprendizaje y que no se puede separar de su contexto.

Nada ajeno al cambio y al poder es la gestión del conocimiento KM; Para Arboníes y Aldazábal (2005), la KM es la activación del conocimiento en actos, rutinas creativas y comunidades que dan sentido a la información. No es la gestión de contenidos, sino la de su flujo en actos relacionales desde una perspectiva de práctica social; es un cambio cultural que exige nuevos roles de los directivos, las personas y en especial de las organizaciones que deben convertirse en lugares de aprendizaje permanente y desarrollo personal.

Al igual que el poder, la gestión del conocimiento tiene hoy una influencia fundamental en la cultura organizacional, en el desarrollo social, en los resultados económicos y en la creación del conocimiento organizacional. De hecho "el control del conocimiento y de la información, genera poder dentro de las organizaciones" (Zapata, et al., 2009b, p.237).

Hoy la economía basada en el conocimiento se fundamenta en el uso de ideas más que en el de capacidades físicas, así como en la aplicación de la tecnología, más que en la transformación de materias primas o la explotación de mano de obra económica. Se trata de una economía en la que el conocimiento es creado, adquirido, transmitido o utilizado más eficazmente por las personas a nivel individual, empresas, organizaciones y comunidades en general para fomentar el desarrollo económico y social (Banco Mundial, 2003).

\section{EL ROL DEL PODER Y LA GESTIÓN DEL EN EL CAMBIO ORGANIZACIO- NAL}

$\mathbb{W} \begin{aligned} & \text { ás vigente que nunca, el pensamiento de } \\ & \text { Foucault acerca del poder parece cobrar vi- }\end{aligned}$ da, cuando en los textos de autores contemporáneos, aparecen de manera coincidente sus ideas. Y es que su pensamiento se proyectó de tal forma que no es fácil quebrar los límites de la frontera del conocimiento en este campo; sin embargo, el acelerado cambio del conocimiento, la tecnología y la innovación, parecen aliarse con los autores contemporáneos en esa misiva.

Focault en su obra "El sujeto y el poder" destaco, el consenso y la violencia como elementos claves del ejercicio del poder, así:

a pesar de que el consenso y la violencia son los instrumentos o los resultados, no constituyen el principio o la naturaleza básica del poder. El ejercicio del poder puede producir tanta aceptación como se desee: puede acumular muertos y refugiarse tras las amenazas que pueda imaginar. En sí mismo, el ejercicio del poder no es una violencia a veces oculta; tampoco es un consenso que, implícitamente, se prorroga. Es un conjunto de acciones sobre acciones posibles; opera sobre el campo de posibilidad o se inscribe en el comportamiento de los sujetos actuantes: incita, induce, seduce, facilita o dificulta; amplía o limita, vuelve más o menos probable; de manera extrema, constriñe o prohíbe de modo absoluto; con todo, siempre es una manera de actuar sobre un sujeto actuante o sobre sujetos actuantes, en tanto que actúan o son susceptibles de actuar (Foucault, 1988, p. 15).

De alguna manera, a través de la violencia, o a través del consenso consentido o tolerado, hoy el poder no ha cambiado su esencia, lo que sí ha cambiado, son los medios o expresiones por medio de los cuales se ejerce o hace manifiesto. 
EL PODER Y LA GESTIÓN DEL CONOCIMIENTO: LAS CLAVES DEL CAMBIO ORGANIZACIONAL

A partir de lo mencionado anteriormente acerca de la esencia del conocimiento, a continuación, se describen y analizan de manera crítica, las principales tendencias derivadas del rol del poder.

En ese orden de ideas, González (2004) describe los retos que genera la rápida disminución de la vida del conocimiento. Este autor menciona que uno de los factores más persuasivos es la reducción de la vida media del conocimiento, es el lapso de tiempo que transcurre entre el momento en el que el conocimiento es adquirido y el momento en el que se vuelve obsoleto.

Siendo así los individuos y las organizaciones poseedores de conocimiento innovador, tienen el privilegio de darle al conocimiento un uso amañado o a conveniencia; es decir, el conocimiento puede ser utilizado en la cantidad, calidad y grado de oportunidad (temporalidad) deseado, según los intereses de los individuos o los grupos y en general toda la organización.

Según Morgan, (1995a) muchos hábiles políticos en la organización ponen su dictamen en práctica, sobre las bases diarias, controlando el flujo de información y el conocimiento que se halla a disposición de la gente, influyendo de ese modo la percepción de las situaciones, y de ahí las maneras en que actúan con relación a esas situaciones. Estos políticos suelen ser conocidos como porteros, abriendo y cerrando canales de comunicación y filtrando, resumiendo, analizando y modelando así el conocimiento de acuerdo con una visión del mundo que favorece sus intereses.

Imbuido en el campo del conocimiento, está el poder de la tecnología; éste proporciona a sus usuarios la capacidad y el control para lograr resultados sorprendentes en una actividad productiva, y también la habilidad de manipular su poder productivo $\mathrm{y}$ hacerlo trabajar eficientemente para sus propios fines. (...) La tecnología diseñada para controlar y dirigir el trabajo de los empleados llega a ser frecuentemente una herramienta de control obrero
(Morgan, 1995b).

En la misma línea del conocimiento y la tecnología, las organizaciones que ostentan además, la capacidad de innovar, tienen el privilegio de controlar a su antojo la información a ser difundida. Debido a la imposibilidad de ser imitados rápidamente, el poder que otorga la innovación, y la frecuencia con que se aplique, permite a quienes lo poseen, programar la obsolescencia y valor de los tangibles y los intangibles; en otras palabras quien controla la innovación (y la tecnología) determina en gran medida la vigencia del valor de un bien, y su obsolescencia.

En síntesis, el cambio organizacional es fuertemente influenciado por el poder del conocimiento, la tecnología y la innovación. El cosmos en medio del cual se mueven las organizaciones ya no es el mismo, el control de fuerzas internas y externas a las organizaciones moldean continuamente la cultura corporativa.

Es necesario a esta altura, distinguir las relaciones de poder de las relaciones de comunicación que transmiten una información por medio de un lenguaje, un sistema de signos o cualquier otro medio simbólico. La comunicación es siempre, sin duda, una cierta manera de actuar sobre el otro o los otros. Pero la producción y la circulación de elementos del significado pueden tener como objetivo o como consecuencia ciertos efectos de poder; estos últimos no son simplemente un aspecto de las primeras (Foucault, 1988, p.12).

Ahora bien, el complejo tejido de comunicación, conocimiento y poder, es esgrimido como uno de los principales factores determinantes del cambio; Sin embargo es importante recordar que el cambio organizacional trae consigo, la resistencia y el conflicto.

Además "Cuando el cambio envuelve la modificación o ampliación de conceptos intelectuales, se supone, generalmente con razón, que indicar a una persona sus áreas deficientes, o sus fallas en las mismas, es suficiente para inducirla a cambiar y a 
EL PODER Y LA GESTIÓN DEL CONOCIMIENTO: LAS CLAVES DEL CAMBIO ORGANIZACIONAL

aceptar la ayuda y recomendación de alguien que, ante sus ojos, tenga la competencia técnica para guiar ese cambio" (Infante, 1996, p. 25).

En la búsqueda de la creación y la transferencia de conocimiento, Nonaka y Takeuchi (1999a) mencionan que su modelo dinámico de creación de conocimiento se fundamenta en el supuesto crítico de que el conocimiento humano se crea y expande a través de la interacción social de conocimiento tácito y de conocimiento explícito. Interacción a la cual ellos llaman conversión de conocimiento. Ellos, postularon cuatro formas de conversión de conocimiento: (a) De tácito a tácito (Socialización); (b) De tácito a explícito (exteriorización); (c) De explícito a explícito (combinación); y, (c) De explícito a tácito (interiorización).

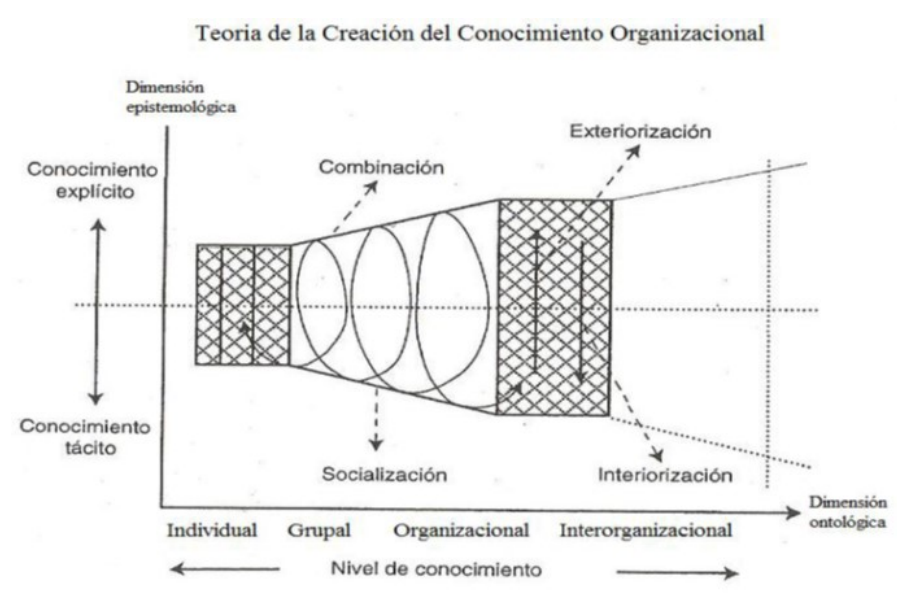

Fuente: Nonaka y Takeuchi (1999b, p.83)

Se comprende entonces que en la medida en que el conocimiento se incrementa sinérgicamente, y pasa de tácito a explícito, y de individual a grupal, organizacional e interorganizacional, genera una espiral de cambio planeado que se fortalece mientras crece en medio de una interacción desarrollada en redes interdisciplinarias. Este ambiente se torna cada vez más dinámico debido a la naturaleza variable del entorno hoy.

"Debido a los ambientes cada vez más dinámicos, las organizaciones son confrontadas en forma conti- nua con la necesidad de implementar cambios en su estrategia, estructura, proceso y cultura" (French, Bell Jr., Zawacki, 2007, p.260). El ejercicio del poder en ambientes dinámicos debe establecer reglas claras y transparentes que permitan la gobernabilidad. "En particular, la comprensión del poder en los recursos, procesos y significados es necesario para alinear los cambios en la estructura, sistemas, personas y cultura" (Dhillon, 2003, p. 643).

Se identifican entonces dos elementos estratégicos para el cambio. Por un lado está la gestión del conocimiento $\mathrm{KM}$, la cual no sólo está ayudando a cerrar las brechas entre el conocimiento tácito y el conocimiento explicito, sino que además está facilitando la creación de ambientes que contribuyen a disminuir el conflicto organizacional.

El otro elemento estratégico facilitante del cambio, es la armonización entre poder y política. Aunque la relación entre los conceptos de poder y política han sido ampliamente estudiados, (Block, 1987; Buchanan y Badham, 1999, 2008; Drory, 1993; Drory y Romm, 1988; Kurchner-Hawkins y Miller, 2006; Lewis, 2002; Mintzberg, 1983) aun no existe suficiente claridad por parte de los investigadores acerca de los aspectos específicos, ni los niveles de análisis de tal vinculo (Landells y Albrecht, 2013).

Sin embargo, el establecimiento claro de políticas facilita la estabilidad del clima político organizacional, lo cual contribuye con la gobernabilidad y la institucionalidad.

Landells y Albrecht (2013) sostienen que las políticas de la organización pueden ser conceptualizadas como un constructo de nivel climático multidimensional; Estos autores propusieron un modelo de cuatro dimensiones denominado el modelo del clima político de la organización, el cual se sustenta en perspectivas teóricas existentes acerca del concepto de poder. En el modelo destacan cuatro dimensiones clave: la construcción y el uso del poder personal, el poder posicional, el poder de conexión y poder informativo. 
EL PODER Y LA GESTIÓN DEL CONOCIMIENTO: LAS CLAVES DEL CAMBIO ORGANIZACIONAL

Ahora bien, existe una clara conexión entre el poder y el conocimiento. Ya lo mencionara Francis Bacon, cuando decía: "conocimiento es poder, no mero argumento u ornamento. El conocimiento y el poder son la misma cosa, pues cuando la causa no se conoce, el efecto no se produce. Para dominar la naturaleza es preciso obedecerla... la sutilidad de la naturaleza es mucho mayor que la sutilidad de los sentidos y la comprensión" (Valhondo, 2010, p.6).

Existentes en la teoría administrativa y la gestión del conocimiento, son muchas las atribuciones que se le dan a la KM en relación al poder. Entre ellas se encuentran varias de las facilidades que tienen $\mathrm{u}$ otorgan el poder de conexión y el poder informativo, cuando se habla del concepto de red, conectivismo, comunidades de practica $(\mathrm{CoP})$, y rutinas creativas entre otros. Estos conceptos están transformando las organizaciones.

Se observan algunos de ellos a continuación. En la actualidad, la organización del conocimiento consiste en "redes" dinámicas y flexibles y en modelos capaces de reacción y adaptación a los cambios. De ahí se deriva la importancia de aprender y conocer, como procesos basados en conexión, puesto que hoy quien trabaja con el conocimiento, necesariamente debe estar familiarizado con los procesos de aprendizaje en red. Por tanto, el cometido esencial de la educación para promover la construcción de entornos de aprendizaje enriquecidos por las redes, ha comenzado ya (Siemens, 2007a).

Las organizaciones que no interactúan en red están corriendo el riesgo de desaparecer, no sólo por que pierden conexión con las demás organizaciones, sino porque disminuyen la posibilidad de desarrollar el conocimiento que surge de las relaciones, o conocimiento relacional. Este conocimiento está siendo enriquecido, por la sinergia y cooperación de capacidades empresariales.

El conectivismo es la integración de principios explorados por las teorías de caos, redes, complejidad y auto-organización. El aprendizaje en ese contex- to, es un proceso que ocurre al interior de ambientes difusos de elementos centrales cambiantes - que no están por completo bajo control del individuo. El aprendizaje, definido como conocimiento aplicable, puede residir fuera de nosotros al interior de una organización o una base de datos, está enfocado en conectar conjuntos de información especializada, y las conexiones que nos permiten aprender más, y tienen mayor importancia que nuestro estado actual de conocimiento (Siemens, 2007b).

Dado que la empresa moderna está abocada al permanente cambio, y además, teniendo en cuenta que la innovación ha cobrado un valor fundamental, las organizaciones deben preocuparse por permanecer vigentes, en términos de la selección de sus estrategias, su alineación y su configuración.

Los párrafos anteriores muestran que el conectivismo visto como un poder juegua un papel fundamental en la flexibilidad, y la adaptabilidad de las organizaciones a las circunstancias del entorno, y por lo tanto, las hace más competitivas.

En la actual sociedad, denominada la sociedad del conocimiento, las organizaciones tienen que ajustar sus prácticas a las nuevas características y contextos del conocimiento. Los métodos de actuación jerárquicos y verticales no están en disposición de reaccionar adecuadamente ni de adaptarse a los cambios con suficiente rapidez. La educación tradicional está al servicio de una visión del aprendizaje como transmisión del conocimiento y como algo estático. Los elementos que ayer eran fundamentales, están dando paso a estructuras descentralizadas y en red. La era digital cambia todo, desde la producción y el consumo de los medios, hasta la forma en que estructuramos el liderazgo, pasando por el modo en que organizamos y gestionamos la administración, la academia y la investigación (Siemens, 2007c). 
EL PODER Y LA GESTIÓN DEL CONOCIMIENTO: LAS CLAVES DEL CAMBIO ORGANIZACIONAL

Las comunidades de práctica PCo - También llamadas Comunidades de Aprendizaje, se constituyen en unidades básicas para construir las estrategias de innovación en una organización. Son un conjunto de personas organizadas alrededor de intereses $o$ competencias comunes que surgen de forma natural en las organizaciones para crear, compartir, aplicar y explotar el conocimiento al interior de los grupos, equipos y unidades. Es un grupo social articulado alrededor de una práctica que genera un sentido comunitario y de identidad, donde se comparte, crea y fluye el conocimiento. Para Arboníes et al. (2007a), las CoP presentan un ciclo de vida que está conducido y marcado básicamente por el interés común del grupo de personas que la componen. Las CoP emergen, se desarrollan, maduran y desaparecen.

Otro de los elementos claves en la KM, son las rutinas creativas; éstas, son rutinas muy específicas que parten de la base de la utilización de la inteligencia de las personas o participantes en una reunión. La creatividad es un hábito en estas reuniones. Según Arboníes et al. (2007b), son rutinas de alto orden, de mayor rango que las rutinas de mejora, donde se toman decisiones de carácter organizacional. Estas rutinas son acogidas en comunidades de práctica y constituyen parte esencial del proceso creativo en un Organización del Conocimiento.

Esto muestra, en resumen, que la KM, y sus diferentes manifestaciones elementos constitutivos, están movilizando el conocimiento de forma permanente, desde una frontera a otra superior o diferente, lo cual otorga ventajas a las organizaciones que las utilizan inteligentemente.

En complemento se describen a continuación otros de los elementos asociados a la KM, que cristalizan la base el conocimiento en este campo y que asociados a la transformación organizacional, están generando poder y cambio.

Bricall (2000) afirma que "conocimiento, innovación y capacidad de aprendizaje son los tres aspectos complementarios del desenvolvimiento actual de las sociedades avanzadas" (p.96); según Bueno (2003) "tríada que enmarca de una forma dinámica y recurrente los procesos de creación y dirección de conocimiento con los de capital intelectual y los de aprendizaje organizativo" (p.19). Se observa como desde la perspectiva de los autores, la innovación, el conocimiento, y la permanente disposición al aprendizaje, constituyen hoy una competencia que lleva a las organizaciones estados de revitalización y competitividad.

El capital intelectual se reconoce hoy como uno de los activos de mayor movilidad en la economía moderna. El conocimiento como activo intangible se constituye en centro de atención de todo tipo de organizaciones, pues éste, administrado eficazmente se ha convertido en el recurso estratégico que jalona la transformación de las organizaciones y la sociedad misma creando riqueza y generando valor (Santamaría, 2011, p. 52).

Volkoff, Elmes, y Strong (2005) afirman que una $\mathrm{CoP}$ intermedia surge en el contexto (sistémico empresarial) porque no existe un propósito común, ni unas prácticas comunes. El tránsito de las organizaciones hacia el cambio necesariamente tiene que ver con el mejoramiento del clima organizacional, para lo cual es necesario tener claras las relaciones o vínculos de poder establecidos entre las personas de la organización. Dependiendo del tipo de poder ejercido, las personas desarrollan relaciones de dominación y aceptación del dominio. Estas relaciones se asocian con el hecho de que las personas son capaces de establecer acuerdos políticos en medio de los cuales pueden cumplir sus objetivos personales y grupales y organizacionales. 
EL PODER Y LA GESTIÓN DEL CONOCIMIENTO: LAS CLAVES DEL CAMBIO ORGANIZACIONAL

\section{CONCLUSION}

$\mathrm{D}$ ebido al itinerante cambio del conocimiento, la gestión del conocimiento ha revolucionado el proceso de cambio en las Organizaciones. Nuevas dimensiones han permeado la capacidad la cultura organizacional permitiendo desarrollar el nuevos poderes organizacionales que hoy día son tendencia.

Entre estas dimensiones se menciona el poder de las redes de conocimiento, el conectivismo, las comunidades de práctica, y las rutinas creativas. Estás han permitido transformar las configuraciones organizacionales, y hoy son tendencias explicitas, que aumentan la competitidad de las organizaciones.

Las nuevas configuraciones empresariales, innovan los procesos, los productos, incluso modifican el mercado. Esta complejidad hace que las organizaciones estén dispuestas para encontrar nuevos escenarios de competencia, incluso distintos a los existentes. En este sentido, la amplia gama acciones que pueden aplicarse en las organizaciones, desde el poder del conocimiento, se constituyen en una capacidad fundamental a la hora de generar valor.

Desde esta óptica no sólo es importante distinguir los diferentes tipos de poder, sino también reconocer el papel que juega la gestión del conocimiento como facilitador de vínculos personales y grupales interorganizacionales o en red.

La adecuación de modelos de gestión de conocimiento se ha convertido en un mecanismo propicio para el desarrollo de las organizaciones, toda vez que los vínculos de dominación y poder están siendo sustentados en el desarrollo y creación del conocimiento producto de un proceso gradual de adaptación de conocimiento tácito en conocimiento explicito.

Las relaciones de poder son políticamente negociadas, lo cual no solamente contribuye con el mejora- miento del clima político de la organización, sino con su transformación hacia un cambio mediado con ayuda de la gestión del conocimiento.

Sumado a esto está el hecho de que las redes de personas, o redes de conocimiento cuentan con un factor "catalizador" del cambio, como lo son las tecnologías de la información y la comunicación (TIC), y un tanto más allá, existe un "tejido vital" que se reconfigura permanentemente, las redes sociales interorganizacionales y especializadas.

Hoy es claro que gracias al conocimiento, la gestión del conocimiento, y el poder que otorgan estos, rápidamente están mutando los bienes, las organizaciones y los mercados en medio de umbrales innovadores e insospechados. 
EL PODER Y LA GESTIÓN DEL CONOCIMIENTO: LAS CLAVES DEL CAMBIO ORGANIZACIONAL

\section{REFERENCIAS}

Agyris, C. ( 1999). Conocimiento para la acción. Una guía para superar los obstáculos para el cambio en la organización. Barcelona: Ed. Granica.

Arboníes, A. L. \& Aldazabal, J. (2005). K-FACTS: Identificación y evaluación de flujos de conocimiento en las organizaciones. Arrasate, GipuzkoaEspaña: Mik, S.Coop

Arboníes, A. L., Aldazabal, J., Arregi, B., Calzada, I., Fínez, J. y Lizaso, I. (2007). Rutinas creativas. Dinámica en equipos de alto rendimiento. Arrasate, Gipuzkoa- España: MIK, S.Coop.

Banco Mundial (2003). Aprendizaje permanente en la economía global del conocimiento: Informe del Banco Mundial. México: Alfaomega.

Block, P. (1987). The empowered manager: Positive political skills at work. San Francisco: JosseyBass Publishers.

Bricall, J. (2000). Informe Universidad 2000 (Bricall). Un periodo de transición en la Universidad. Recuperado de http://www.uam.es/ presentacion/univ2000/cap1.pdf

Buchanan, D., \& Badham, R. (1999). Power, politics, and organizational change: Winning the turf game. London: Sage Publications.

Buchanan, D., \& Badham, R. (2008). Power, politics and organizational change: Winning the turf game. London: Sage Publications.

Bueno, E. (2003). Gestión del Conocimiento en Universidades y Organismos Públicos de Investigación. (Universidad Autónoma de Madrid). Recuperado de http://www.madrimasd.org/informacionidi/ biblioteca/publicacion/doc/16 mientoUniversidadesOPIS.pdf
Dhillon, G. (2003). Dimensions of power and IS implementation. Information \& Management 41 (2004) pp. 635-644

Drory, A. (1993). Perceived political climate and job attitudes. Organization Studies, 14 (1), 59-71.

Drory, A., \& Romm, T. (1988). Politics in organization and its perception within the organization. Organization Studies, 9 (2), 165-179.

Foucault, M. (1988). El sujeto y el poder. Revista Mexicana de Sociología, Vol. 50, No. 3. pp. 3-20. Tomado de http://www.jstor.org/ discover/10.2307/3540551?uid $=3737808$

\&uid $=2134 \&$ uid $=2 \&$ uid $=70 \&$ uid $=4 \&$ sid $=2110362$ 1348257

French, W., Bell, C. \& Zawacki, R. (2007). Desarrollo organizacional: transformación y administración efectiva del cambio. Parte 5. Lineamientos y temas de Implementación. Lectura 28. Crear disposición para el cambio. España: McGraw-Hill Interamericana.

González, C. (2004). The Role of Blended Learning in the World of Technology. Benchmarks Online. Recuperado de http://www.unt.edu/benchmarks/ archives/2004/september04/eis.htm

Infante, A. (1996). Desarrollo Organizacional. Una nueva manera de guiar el trabajo de los hombres. Facultad de Admininstración. Monografía No. (44). Bogotá: Tercer Mundo. Editores. P. 25.

Kurchner-Hawkins, R., \& Miller, R. (2006). Organizational politics: Building positive political strategies in turbulent times. In E. Vigoda-Gadot, \& A. Drory (Eds.), Handbook of organizational politics (pp. 328-352). Cheltenham, UK: Edward Elgar.

Landells, E., Albrecht, S. (2013). Organizational political climate: Shared perceptions about the building and use of power bases. Original Research Article Human Resource Management Review, Volume 23, Issue 4, pp. 357-365. 
EL PODER Y LA GESTIÓN DEL CONOCIMIENTO: LAS CLAVES DEL CAMBIO ORGANIZACIONAL

Lewis, D. (2002). The place of organizational politics in strategic change. Strategic Change, 11, 2534.

Li, Bing., Zhang, Jianpeng., Zhang, Xiaoxia. ( 2013 ). Knowledge Management And Organizational Culture: An Exploratory Study. Creative and Knowledge Society. Volume 3, Issue 1, Pages 6577. Recuperado de http://www.degruyter.com/view/ $\mathrm{j} / \mathrm{cks}$.2013.3.issue-1/v10212-011-0031-3/v10212011-0031-3.xml

Mintzberg, H. (1983). Power in and around organizations. Englewood Cliffs, N.J.: Prentice-Hall.

Morgan, G. (1995a). Imagenes de la Organización. México. CAP. 6: intereses, conflicto y poder: Las organizaciones como sistemas políticos. Alfaomega Grupo Editor, S.A. De C.V. MéxicoD.F. (pp. 127 186).

Morgan, G. (1995b). Imagenes de la Organización. México. CAP. 6: intereses, conflicto y poder: Las organizaciones como sistemas políticos. Alfaomega Grupo Editor, S.A. De C.V. MéxicoD.F. (pp. 127 186).

Perry, Milly., Snunith, Shoham. (2009). Knowledge management as a mechanism for technological and organizational change management in Israeli universities. Higher Education, Vol. 57, No. 2. pp. 227-246. Published by: SpringerStable Recuperado de http://www.jstor.org/stable/40269118

Robbins, S. (1998). Fundamentos de comportamiento organizacional. México. Prentice Hall- Hispanoamericana, S.A.

Robbins, S., Judge. T. (2013a). Comportamiento Organizacional. México.: Pearson. p.581 ed. (15).

Robbins, S., Judge. T. (2013b). Comportamiento Organizacional. México.: Pearson. p.581 ed. (15).

Robyn Thomas., Leisa, Sargent., Cynthia, Hardy. (2011). Managing Organizational Change: Negotiating Meaning and Power-Resistance Relations. Organization Science22.1 : 22-41. Recupera- do de http://ezproxy.uninorte.edu.co:2538/ docview $/ 853056949$ ? accountid $=41515$

Sanchez, R., Mahoney, J. (1996). Modularity, Flexibility, and Knowledge Management in Product and Organization Design. Strategic Management Journal, Vol. 17, Special Issue: Knowledge and the Firm(Winter, 1996), pp. 63-76. Published by: WileyStable URL: http://www.jstor.org/ stable/2486991

Santamaría, A. (2010). Gestión del conocimiento en la Universidad de Sucre en Colombia: análisis del sistema de investigación y extensión. Tomado de www.edunexos.edu.co

Siemens, G. (2007a). Conectivismo: Una teoría de aprendizaje para la era digital. Recuperado de http://rpnp2010.comunidadviable.cl/media/ users/3/190432/files/27518/Conectivismo.pdf

Siemens, G. (2007b). Conectivismo: Una teoría de aprendizaje para la era digital. Recuperado de http://rpnp2010.comunidadviable.cl/media/ users/3/190432/files/27518/Conectivismo.pdf

Siemens, G. (2007c). Conectivismo: Una teoría de aprendizaje para la era digital. Recuperado de http://rpnp2010.comunidadviable.cl/media/ users/3/190432/files/27518/Conectivismo.pdf

Sveiby, K. E. (2000). Capital Intelectual: La Nueva Riqueza de las Empresas. Paris: Maxima Laurent du Mesnil.

Thomas, Robyn; Sargent, Leisa D; and Hardy, Cynthia. (2011). Managing Organizational Change: Negotiating Meaning and Power-Resistance Relations.

Organization Science, Vol. 22, No. 1 (JanuaryFebruary 2011) , pp. 22-41 Published by: INFORMS Article Stable URL: http:// www.jstor.org/stable/20868845 
EL PODER Y LA GESTIÓN DEL CONOCIMIENTO: LAS CLAVES DEL CAMBIO ORGANIZACIONAL

Valhondo, D. (2010). Gestión del conocimiento: Del mito a la realidad. Madrid, España. Diaz de Santos. P.6.

Volkoff, O., Elmes, M.B., Strong, D.M. (2005). Enterprise systems, knowledge transfer and power users. Journal of Strategic Information Systems 13 (2004) pp.279-304.

Whittington, R. (2002). ¿Qué es la estrategia? ¿Realmente importa? España. Thomson.

Zapata, Á. et al. (2009a). Teorías contemporáneas de la organización y del Management. Bogotá.: Ecoe Ediciones. Universidad del Valle.

Zapata, Á. et al. (2009b). Teorías contemporáneas de la organización y del Management. Bogotá: Ecoe Ediciones. Universidad del Valle. 